\title{
Spatial and Temporal Expression of High Mobility Group Nucleosome-Binding (HMGN) Genes in Brain Areas Associated With Cognition in Individuals With Down Syndrome
}

\author{
Alejandra Rodriguez-Ortiz \\ Universidad del Valle. Cali \\ Julio Cesar Montoya-Villegas \\ Universidad del Valle. Cali \\ Felipe García-Vallejo \\ Universidad del Valle. Cali \\ Yecid Mina-Paz ( $\square$ yecid.mina@correounivalle.edu.co) \\ Institución Universitaria Escuela Nacional del Deporte
}

\section{Research Article}

Keywords: Down syndrome, Gene expression, Epigenetics, Human brain, HMGN, Neuroinformatics

Posted Date: August 11th, 2021

DOI: https://doi.org/10.21203/rs.3.rs-784407/v1

License: (c) (i) This work is licensed under a Creative Commons Attribution 4.0 International License.

Read Full License

Version of Record: A version of this preprint was published at Genes on December 17th, 2021. See the published version at https://doi.org/10.3390/genes12122000. 


\section{Abstract}

Background: DNA methylation and histone posttranslational modifications are epigenetics processes which contribute to neurophenotype of Down Syndrome (DS). Previous reports present strong evidence that nonhistone High mobility group N proteins (HMGN) are epigenetic regulators. They play important functions in several process to maintain the brain homeostasis. We aimed to analyze the differential expression of five human HMGN genes along some brain structures and age ranks from DS postmorten brain samples.

Methodology: We performed a computational analysis of human HMGN expression from a DNA microarray experiment data (GEO database ID GSE59630). Using the transformed log2 data, we analyzed the differential expression of five HMGN genes in several brain areas associated with cognition in patients with DS. Moreover, using information from several genome databases, we explore the coexpression and protein interactions with the histones of nucleosome core particle and linker $\mathrm{H} 1$ histone.

Results: We registered that HMGN1 and HMGN5 were significantly overexpressed in hippocampus and areas of prefrontal cortex including DFC, OFC and VFC of DS patients. Age ranks comparisons between euploid control and DS individuals showed that HMGN2 and HMGN4 were overexpressed in the DS brain of 16 to 22 weeks of gestation. From BioGRID database we registered high interaction scores of HMGN2 and HMGN4 with Hist1H1A and Hist1H2BA, Hist2AG, and Hist1H3A respectively.

Conclusions: Overall our results give strong evidence to propose that DS would be an epigenetics-based aneuploidy. Remodeling the brain chromatin by HMGN1 and HMGN5 would essential pathway in the modification of brain homeostasis in DS.

\section{Introduction}

The continuous chromatin modification and the binding of tissue specific transcription factors to their specific targets in chromatin, maintain the epigenetic landscape necessary to regulate the cell-type specific transcription [1] [2]. However, additional chromatin modifiers, including the H1 linker histones [2] and the high mobility group N (HMGN) proteins, can remodel the chromatin organization and transcription regulation, playing important functions in several process to maintain the general homeostasis [3]. Down syndrome (DS), is a chromosomal aneuploidy caused by a total or partial triplication of chromosome 21, but in rare cases it can be associated with a process of chromosome translocation [4]. In persons with DS the gene dose imbalance by triplication of genes on HSA21 is mostly associated with a wide spectrum of pathologies that include neurological and systemic diseases [4]. The incidence of trisomy 21 is influenced by maternal age and differs in population [5] [6]. In developed countries, the average life span for DS population is 55 years [7]. Although DS is the result of the increased copy number of a single 21 chromosome, the regulation of gene expression is affected at a genome-wide level [8-11]. 
There is an increasing line of evidence to propose that beyond of HSA21 trisomy, DS is an epigeneticsbased syndrome [12]. For instance, a previous study with fetal skin fibroblasts from a set of monozygotic twins, revealed regions that were predominantly hypermethylated in DS in genes involved in embryonic organ morphogenesis. Reprogramming of the DS fibroblasts to induced pluripotent stem cells (iPSCs), showed that these regions were maintained in the pluripotent state and correlated with differential gene expression and increased expression of the DNA methyltransferases DNMT3B and DNMT3L [13]. Thus, the genome-wide differences seen in DS tissues are correlated with epigenetic modifications would be responsible, in part, for the establishment and/or maintenance of differential expression of genes in and outside of HSA21 in DS

HMGN proteins are a nonhistone protein family that include 5 members encoded by 5 specific genes with a similar intron-exon organization that are localized along human genome in different chromosome loci (Table 1) [13-18]. Previous reports show that HMGN proteins are the only nuclear proteins known to specifically recognize the generic structural features of the 147 base pair nucleosome core particles. In vitro analyses showed that at low ionic strength nucleosome core particles can bind to HMGN proteins with high affinity [19-21]. The interaction of HMGN proteins with nucleosomes is dynamic and the proteins compete with the linker histone $\mathrm{H} 1$ chromatin binding sites [22-24].

Since the role of high mobility group $\mathrm{N}$ proteins (HMGN) as important molecules that reshape the organization of chromatin and transcription levels, we perform a bioinformatic simulation analysis of its spatial and temporal expression in several areas of the brain with Down's Syndrome. To perform these analyses, we obtained log2 data from a free access microarray experiments previously consigned in the GEO DataSets set of NCBI (https://www.ncbi.nlm.nih.gov/). To explore the differential brain expression of HMGN genes, we calculate the Z-ratio from DS brain postmorten samples associated with cognitive processes in those brain areas previously described by Olmos-Serrano et al, 2016 [25]. Our results gave strong evidence to support the hypothesis of the crucial role of non-histones HMGN-1 and HMGN-5 proteins as important spatial and temporal remodelers that would change, by epigenetic process, the brain proteostasis in patients with Down syndrome.

\section{Methodology}

\section{Data Mining}

Raw gene expression data of Down syndrome samples and normal samples were downloaded from the Gene Expression Omnibus (GEO) (http://www.ncbi.nlm.nih.gov/geo/) of the National Center for Biotechnology Information (NCBI). For the analyses performed in the present study, we selected the human genes encoding for the five high mobility group N (HMGN) proteins previously consigned in the Gene Entrez of the NCBI database (https://www.ncbi.nlm.nih.gov/gene), (table 1). Moreover, for all calculations we used the log2 transformed expression values of free a access DNA microarray experiment whose registration code in the GEO database is GSE59630 
(http://www.ncbi.nlm.nih.gov/geo/query/acc.cgi?acc=GSE59630), previously deposited by OlmosSerrano et al. 2016. [25].

According with information consigned in the GEO database, the selected microarray experiment included gene expression data of more than 17,000 probes from 58 post-mortem brain samples of DS patients ( 25 from females and 33 from males) and 58 euploid samples as normal controls ( 25 from females and 33 from males), that were classified by gender, age and also by some brain areas including the hippocampus (HIP), cerebellar cortex (CBC), dorsolateral prefrontal cortex (DFC), orbital prefrontal cortex (OFC), ventrolateral prefrontal cortex (VFC), medial prefrontal cortex (MFC), primary somatosensory cortex (S1C), inferior parietal cortex (IPC), primary visual cortex (V1C), superior temporal cortex (STC), Inferior temporal cortex (ITC). Nevertheless, for the present study we decided to analyze, not only the brain as a whole but also OFC, MFC, HIP and CBC brain regions which are highly associated to neurophenotype of Down syndrome.

\section{Data preprocessing}

The robust multiarray analysis (RMA) algorithm [30] in Affymetrix Power Tools (APT; http://www.affymetrix.com/) was applied to perform background correction and standardization for all raw data, aiming to filter false-positive data. The applied criterion was as follows: at least half the samples had PLIER signal intensity values greater than 100. [27].

\section{Quantification of the differential HMGN genes expression}

Raw intensity log2 data of each experiment were used for the calculation of Z-score [28]. Z-scores of the protein coding genes analyzed, were calculated according to the equation (1):

$$
Z-\text { score }=\frac{(\text { Log intensity of } G-\text { mean } \log \text { intensity } G \ldots G n)}{\text { Standard Deviation } \log G \ldots G n}
$$

\section{Equation 1. Z-score formula}

All Z-score values were normalized on a linear scale $-3.0 \leq 0 \geq+3.0$ (two-tailed $P$ value $<0.001$ ). From Zscore data we calculated the mean values per gene and per structure in brain samples of DS and euploid controls. This data was used to calculate the Z-ratio (Equation 2) with is a measure to estimate differential gene expression; genes with values over 1.96 are considered over-expressed [28].

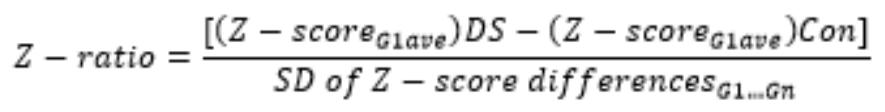

Equation 2. Z-ratio formula

\section{Gene-dosage imbalanced quantification}


To find out the gene dosage imbalance of that HSA21 genes that coexpressed with the five HMGN genes along the cerebral structures of DS brain samples, first we calculate the M values according to the equation 2.1, and then we used the $M$ value to calculate the ratio of the dosage imbalance $R$ (DS Control ratio) as shown in Equations 2.1 and 2.2 [29].

Equation 2.1 M-value formula

$M=\left[\right.$ Mean $\log _{2}(D S)-$ Mean $\log _{2}($ Control $)$

Equation 2.2. R (DS/Control ratio) formula

$R=2^{M}$

$R$ values ranging from 0.80 to 1.30 were considered as normal balanced (two copies of gene); on contrary, if $R$ values ranged $1.4 \leq 1.5 \geq 1.7$, genes are dosage-imbalanced by triplication (three copies per gene), but if $\mathrm{R}$ ratio is greater than 1.8 genes are amplified (more than three copies).

\section{Construction of HMGN genes network using GeneMania}

To build the gene interaction networks of HMGN genes, we applied the free access platform GeneMANIA (http://www.genemania.org) a real-time multiple association network actively developed at the University of Toronto, in the Donnelly Centre for Cellular and Biomolecular Research that use a mssive set of functional association data. [30]. All calculation carried out in the present study were processed using the updated 2018 version

\section{Protein-Protein interaction analysis}

To computational simulate the interaction between each HMGN with several histones of the core particle and $\mathrm{H} 1$, we obtain the data from BioGRID (Database of Protein, Chemical, and Genetic Interactions) a free access database (https://thebiogrid.org/) [31]. BioGRID is an interaction repository with data compiled through comprehensive curation efforts. The current index is version 3.5 and permits to perform searches of 69,922 publications for $1,706,694$ protein and genetic interactions from humans. All data are freely provided via their search index and available for download in standardized formats. The different searches performed in the present study, were from data updated by January of 2019. [31]

\section{Statistical analysis}

For comparing mean values of Z-ratio of DS brain, we performed mulivariated statistical analyses among the different brain cortex structures between DS patients and euploid controls. The $Z$ test/Two-tailed was used to calculate differences in HMGN differential expression. The $p$-values were calculated using the web tool P-value from Z-score Calculator (https://www.socscistatistics.com/pvalues/normaldistribution.aspx). In all cases we use an alfa of 0.05 to test the significance of $\mathrm{H}_{0}$. To calculate the statistical differences in the mean log2 values of DS and 
Controls for gender, age, hippocampus, cerebellar and brain cortex structures, we apply the t-test for two paired samples/Two-tailed test with an alfa of 0.05 .

Principal Component Analysis (PCA), and a Hierarchical Cluster (Heat-map) were employed as a computational procedure for the classification of multiclass gene expression in brain structures between DS and control samples per sex and age. All analyses were run in SPSS program version 25.0 (https://spss.softonic.com/) and Cytoscape 3.6 (https://cytoscape.org/release_notes_3_6_0.html).

\section{Results}

\section{Expression of HMGN genes in brain areas from individuals with DS}

In general, we observed the expression of five HMGN genes was variable along all structures under analysis. Moreover, we recorded significant differences in their overexpression values depending of brain area under analysis. In this sense, the genes encoding for HMGN1 and HMGN5 were overexpressed not only in HIP, CBC and V1C but in some areas of prefrontal cortex including DFC, OFC and VFC (values of Zratio>1.96); in contrast with not significant over expression of HMGN2, HMGN3 genes. Only in ITC HMGN5 gene registered significant Z-ratio (Z-ratio=2.0) (Table 2).

Since the HMGN1 gene is localized at 21q22.2 band, we calculated the level of dose imbalance in those brain structures including in the present study. Our results showed that HMGN1 was dosage imbalanced, in OFC, VFC and CBC by triplication $(R>1.4)$, but in HIP, DFC and ITC was dysbalanced by amplification $(\mathrm{R}>1.8)$.

\section{Age dependent expression of HMGN genes in the brain of individuals with DS}

HMGN4 (Z-ratio=4.72) and HMGN2 (Z-ratio=2.13), were significant overexpressed in prenatal samples of DS brain (16 to 22 weeks of gestation) in comparison with other age ranks (table 3 ). The data of Z-ratio for HMGN3 showed significant overexpression values in the brain of DS during the first year (0-12 months), childhood ( 2 to 10 years), 12 to 22 years old samples, and adulthood (32 to 42 years old) but not in brain samples of prenatal brain samples ( 16 to 22 weeks of gestation; Z-ratio=1.08) (table 3 ). In contrast, the Z-ratio values for HMGN1 and HMGN5 along the different age ranks were non-significant, except for HMGN1 that was overexpressed in rank of 12 to 22 years old (Z-ratio=2.0) (Table 3)

\section{Protein-Protein Interaction Network and GO categories}

The Protein-Protein Interaction (PPI) network made with the five HMGN genes accounted for a total of 73 nodes, two connected components, one multi-edge node pair, an average number of neighbors of 2.374, and a heterogeneity of 2.012 (Figure 1). The node with the highest number of interactions was HMGN1 with 30 , followed closely by HMGN2 with 28 . GO categories of biological processes most relevant of the network included indispensable epigenetic processes for chromatin activation or inactivation such as histone deacetylation (P-value 2.46E-08) and histone H3-K4 methylation (P-value 1.88E-07). - 


\section{HMGN proteins interaction with Histones of nucleosome core and linker H1}

From data of several experimental methods reported in BioGRID data base and consigned in table 4, we observed differential high interaction scores of HMGN proteins with histones of the nucleosome core HIST1H2AG, HIST1H2BA, HIST1H3A and HIST1H4A (table 3). Only HMGN2 has a significant high score of interaction with the linker histone HIST1H1A and the histones of nucleosome core HIST1H3A and HIST1H2AG. HMGN1 and HMGN5 showed significant interaction scores only with HIST1H4A. Finally, HMGN3 interacts with the HIST1H4A

\section{Discussion}

Previously, some studies presented strong evidence that in DS individuals the genome-wide epigenomic alterations occur not only in chromosome 21 but in some other chromosomes [32-34]. these include changes in gene expression, RNA content, and epigenetics histone modifications, nucleosome spacing, and DNA methylation process which are dependent of health status and age [35]. Specifically, there are strong evidences that HMGN proteins play a role in epigenetic regulation of gene expression and play important functions in several biological process to maintain the normal homeostasis and altered gene expression in disease [36-37]. Thus, we aimed to analyze from a bioinformatics approach, the gene expression of human HMGN genes in different human brain structures and age ranks comparing DS brain samples and euploid controls

Most of the literature reports about the expression of HMGNs in the brain as whole and also in neuronal derived cells, come from experiments carried out in mice [38]. In this sense its interpretation could be cautiously extended to humans and also to the brain of Down syndrome. In this scenario, our results are the first to analyze in a representative sample of euploid individuals and Down syndrome patients the differential expression of the five human HMGN in several area of the brain which are involved in learning and memory and also its age ranks variation. Our bioinformatics approach permitted to obtain strong statistical evidence of the differential regulation of HMGNs in the disruption of the normal brain homeostasis in some areas associated with the Down syndrome neurophenotype.

HMGN1 is in a region of human chromosome 21 and it is frequently found triplicated in DS samples. Our results not only confirmed the previous reports but extended the data to the brain of individuals with DS. HMGN1 gene was dose dysbalanced by triplication in the whole brain, and brain cortex, and dysbalanced by amplification in the hippocampus $[39,40]$. The hippocampus is a brain structure that plays a major role in neural plasticity and cognition [39], which is known to the dysregulated in individuals with DS. Our results showed that methyl CpG-binding protein 2 (MeCP2) is underexpressed in several structures of the brain of DS, which can be linked to the dysregulation of the gene HMGN1, given that this latter gene can affect the expression of MeCP2 by changing the chromatin structure and histone modifications in the MeCP2 promoter [40].

According to the PPI network the nodes with the highest number of interactions were HMGN1 and HMGN2. Moreover, the GO categories showed a global implication of these genes in chromatin 
remodeling processes such as acetylation, methylation of histones, and dendritic spine morphogenesis. The dysregulation of gene expression recorded in these genes would most certainly affect the interactions with others, and would possibly leading to the epigenomic changes found in individuals with DS.

Some analysis suggests that HMGNs could differentially modulate no only the global gene transcription in some brain structures but also in other tissues [41, 42]. Therefore, the contribution of HMGN1 and HMGN5 to the transcriptional dysregulation of DS neurophenotype need to be studied separately in specific developmental scenarios. In mice HMGN1 is a negative regulator of the brain expression of Mecp2 (methyl CpG-binding protein 2), that promotes HMGN1 overexpression associated with some effects not only in general behavioral activities but in anxiety and social deficits too [43,44]. On the other hand, HMGN5, is thought to reduce the compactation of the chromatin fiber nucleosomes, thereby enhancing transcription from chromatin templates, nevertheless, it has not been related to DS.

Excluding the gestational period (12-16WG), only HMGN3 had a statistically significant expression across different age-ranks. It contrasts with the expression of HMGN2 and HMGN4 which had significant differential expression in the gestational period but not in the rest of the age-ranks we evaluated. In this sense, our results support some data found in literature that HMGN3 control part some epigenetic mechanisms during the neuronal development [43]. HMGN2 expression has been widely associated with embryogenesis [45]; anti-sense manipulation of HMGN2 gene leads to early embryonic abnormalities [46, 47] Our results suggest that HMGN2 regulates active and bivalent genes by promoting an epigenetic landscape of active histone modifications at promoters and enhancers. The conclusion is that HMGN2 stabilize the epigenetic landscape necessary for maintaining the pluripotent identity of pluripotent stem cell [47].

Overall, our results give strong evidence to propose that DS would be an epigenetics-based aneuploidy and the spatial and temporal differential expression of HMGNs would play important roles in the modification of brain homeostasis in DS. We report the differential interaction of HMGN family proteins with histones of the nucleosome core HIST1H2AG, HIST1H4A, HIST1H2AG, HIST1H3A, HIST2H2AB, HIST1H4A and also with the linker HIST1H1A. In this scenario, we propose that HMGN proteins play an important role in the topological process of remodeling the chromatin in several brain areas of Down's syndrome that are associated with memory and learning process. Global effect of those epigenetics deregulation would be the alteration of the brain homeostasis that potential conditioned the brain in Down's syndrome epigenetics mode.

\section{Declarations}

We certified that have no commercial associations (e.g., consultancies, stock ownership, equity interest, patent/licensing arrangements, etc.) that might pose a conflict of interest in connection with the submitted article, except as disclosed on a separate attachment. All funding sources supporting the work and all institutional or corporate affiliations of the authors are acknowledged in a footnote in the Work 
Funding: No funding was required for this work

Conflicts of interest/Competing interests: None of the authors presents a conflict of interest.

Ethics approval: DNA microarray experiment whose registration code in the GEO database is GSE59630 (http://www.ncbi.nlm.nih.gov/geo/query/acc.cgi?acc=GSE59630).

Consent to participate: DNA microarray experiment whose registration code in the GEO database is GSE59630 (http://www.ncbi.nlm.nih.gov/geo/query/acc.cgi?acc=GSE59630).

Consent for publication: DNA microarray experiment whose registration code in the GEO database is GSE59630 (http://www.ncbi.nlm.nih.gov/geo/query/acc.cgi?acc=GSE59630).

Availability of data and material: DNA microarray experiment whose registration code in the GEO database is GSE59630 (http://www.ncbi.nlm.nih.gov/geo/query/acc.cgi?acc=GSE59630).

Code availability: None

\section{Acknowledgements and Authors' contributions}

Acknowledgements: To the Universidad del Valle and the Molecular Biology and Patogenesis research group

AR-O, FG-V and YM-P searched data from a DNA microarray experiment in SD and found microarray experiment with ID GSE59630 previously deposited in the GEO DataSet of NCBI database. AR-O and YM-P using the transformed log2 data, analyzed the differential expression of five HMGN genes in several brain areas associated with cognition in patients with DS. AR-O, YM-P and JCM explored the coexpression and protein interactions with the histones of nucleosome core particle and linker $\mathrm{H} 1 \mathrm{histone.} \mathrm{All} \mathrm{authors}$ worked on the results and conclusions. Similarly, they read and approved the final manuscript.

\section{References}

1. .Aït Yahya-Graison, E. et al. Classification of human chromosome 21 gene-expression variations in Down syndrome: impact on disease phenotypes. Am J Hum Genet, 81 (3), 475-491 Epub 2007 Jul 19. PubMed PMID: 17701894; PubMed Central PMCID: PMC1950826 (2007).

2. Abuhatzira, L., Shamir, A., Schones, D. E., Schäffer, A. A. \& Bustin, M. The chromatin-binding protein HMGN1 regulates the expression of methyl CpG-binding protein 2 (MECP2) and affects the behavior of mice. J Biol Chem, 286 (49), 42051-42062 https://doi.org/10.1074/jbc.M111.300541 (2011).

3. Antonarakis, S. E. Down syndrome and the complexity of genome dosage imbalance. Nat Rev Genet, 18, 147-163 (2016).

4. Belova, G. I., Postnikov, Y. V., Furusawa, T., Birger, Y. \& Bustin, M. Chromosomal protein HMGN1 enhances the heat shock-induced remodeling of Hsp70 chromatin. J Biol Chem, 283, 8080-8088 (2008). 
5. Breitkreutz, B. J., Stark, C. \& Tyers, M. "The GRID: the General Repository for Interaction Datasets". Genome Biology, 4, R23 https://doi.org/10.1186/gb-2003-4-3-r23 (2003).

6. Canfield, M. A. et al. (2006). National estimates and race/ethnic-specific variation of selected birth defects in the United States, 1999-2001. Birth Defects Res A Clin Mol Teratol.76(11):747 - 56. doi: 10.1002/bdra.20294. PMID: 17051527.

7. Carothers, A. D., Hecht, C. A. \& Hook, E. B. International variation in reported live birth prevalence rates of Down syndrome, adjusted for maternal age. J Med Genet, 36, 386-393 (1999).

8. Cheadle, C., Vawter, M. P., Freed, W. J. \& Becker, K. G. Analysis of microarray data using Z score transformation. The Journal of molecular diagnostics: JMD, 5 (2), 73-81 https://doi.org/10.1016/S1525-1578(10)60455-2 (2003).

9. Chen, T. \& Dent, S. Y. R. Chromatin modifiers and remodellers: regulators of cellular differentiation. Nature Reviews Genetics, 15, 93-106 (2014).

10. Costa, V. et al. Massive-scale RNA-Seq analysis of non ribosomal transcriptome in human trisomy 21. PLoS One, 6 (4), e18493 https://doi.org/10.1371/journal.pone.0018493 (2011).

11. Do, C. et al. Trans-acting epigenetic effects of chromosomal aneuploidies: lessons from Down syndrome and mouse models. Epigenomics, 9, 189-207 (2017).

12. Garza-Manero, S. et al. Maintenance of active chromatin states by HMGN2 is required for stem cell identity in a pluripotent stem cell model. Epigenetics Chromatin. 2019 Dec 12;12(1):73. doi: 10.1186/s13072-019-0320-7.

13. Gensous, N., Franceschi, C., Salvioli, S., Garagnani, P. \& Bacalini, M. G. Down Syndrome, Ageing and Epigenetics. Subcell Biochem, 91, 161-193 https://doi.org/10.1007/978-981-13-3681-2_7 (2019).

14. Glasson, E. J. et al. The changing survival profile of people with Down's syndrome: implications for genetic counselling. Clin Genet, 62, 390-393 https://doi.org/10.1034/j.1399-0004.2002.620506.x (2002).

15. Hochedlinger, K. \& Jaenisch, R. Induced pluripotency and epigenetic reprogramming. Cold Spring Harb. Perspect. Biol, 7, a019448 (2015).

16. Hock, R., Furusawa, T., Ueda, T. \& Bustin, M. HMG chromosomal proteins in development and disease. Trends in Cell Biology, 17 (2), 72-79 (2007).

17. Irizarry, R. A. et al. Exploration, normalization, and summaries of high density oligonucleotide array probe level data. Biostatistics, 4, 249-264 https://doi.org/10.1093/biostatistics/4.2.249 (2003).

18. Ito, Y. \& Bustin, M. Immunohistochemical localization of the nucleosome-binding protein HMGN3 in mouse brain. J. Histochem. Cytochem, 50, 1273-1275 (2002).

19. Kahmann, N. H. \& Rake, A. V. Altered nucleosome spacing associated with Down syndrome. Biochem. Genet, 31, 207-214 (1993).

20. Korner, U., Bustin, M., Scheer, U. \& Hock, R. Developmental role of HMGN proteins in Xenopus laevis. Mech Dev, 120, 1177-1192 (2003). 
21. Kugler, J. E. et al. High mobility group $N$ proteins modulate the fidelity of the cellular transcriptional profile in a tissue- and variant-specific manner. J. Biol. Chem, 288, 16690-16703 (2013).

22. Kugler, J. E., Deng, T. \& Bustin, M. The HMGN family of chromatin-binding proteins: dynamic modulators of epigenetic processes. Biochimica et biophysica acta, 1819 (7), 652-656 https://doi.org/10.1016/j.bbagrm.2012.01.013 (2012).

23. Lane, A. A. et al. Triplication of a 21q22 region contributes to B cell transformation through HMGN1 overexpression and loss of histone H3 Lys27 trimethylation. Nat. Genet, 46, 618-623 (2014).

24. Letourneau, A. et al. Domains of genome-wide gene expression dysregulation in Down's syndrome. Nature, 508, 345-350 (2014).

25. Mao, R. et al. Global up-regulation of chromosome 21 gene expression in the developing Down syndrome brain. Genomics. 2003;81:457-467.

26. Mardian, J. K., Paton, A. E., Bunick, G. J. \& Olins, D. E. Nucleosome cores have two specific binding sites for nonhistone chromosomal proteins HMG 14 and HMG 17., 209, 1534-1536 (1980).

27. Mégarbané, A. et al. The 50th anniversary of the discovery of trisomy 21: the past, present, and future of research and treatment of Down syndrome. Genet Med, 11, 611-616 https://doi.org/10.1097/GIM.0b013e3181b2e34c (2009).

28. Mohamed, O. A., Bustin, M. \& Clarke, H. J. High-mobility group proteins 14 and 17 maintain the timing of early embryonic development in the mouse. Dev Biol, 229, 237-249 (2001).

29. Mostafavi, S., Ray, D., Warde-Farley, D., Grouios, C. \& Morris, Q. GeneMANIA: a real-time multiple association network integration algorithm for predicting gene function. Genome biology, 9 (Suppl 1(Suppl 1), S4 https://doi.org/10.1186/gb-2008-9-s1-s4 (2008).

30. Nanduri, R., Furusawa, T. \& Bustin, M. Biological Functions of HMGN Chromosomal Proteins. Int. J. Mol. Sci, 21 (2), 449 https://doi.org/10.3390/ijms21020449 (2020).

31. Olmos-Serrano, J. L. et al. Down Syndrome Developmental Brain Transcriptome Reveals Defective Oligodendrocyte Differentiation and Myelination.Neuron. 2016 Mar16;89(6):1208-1222. doi: 10.1016/j.neuron.2016.01.042. Epub 2016 Feb 25. PubMed PMID: 26924435; PubMed Central PMCID: PMC4795969.

32. Oughtred, R. et al. The BioGRID interaction database: 2019 update. Nucleic acids research, 47 (D1), D529-D541 https://doi.org/10.1093/nar/gky1079 (2019).

33. Patil, A. \& Nakamura, H. Disordered domains and high surface charge confer hubs with the ability to interact with multiple proteins in interaction networks. FEBS Lett, 580, 2041-2045 (2006).

34. Patterson, D. Genetic mechanisms involved in the phenotype of Down syndrome. Ment Retard Dev Disabil Res Rev, 13, 199-206 https://doi.org/10.1002/mrdd.20162 (2007).

35. Postnikov, Y. V. et al. Homodimers of chromosomal proteins HMG-14 and HMG-17 in nucleosome cores. J Mol Biol. 1995;252:423-432.]., Histone methylation versus histone acetylation: new insights into epigenetic regulation. Curr Opin Cell Biol. 2001;13:263-273. 
36. Rochman, M. et al. The Interaction of NSBP1 With Nucleosomes In Euchromatin Counteracts Linker Histone-Mediated Chromatin Compaction And Modulates The Fidelity Of The Cellular Transcription Profile. Mol Cell, 35, 642-656 (2009).

37. Rubin, R. D., Watson, P. D., Duff, M. C. \& Cohen, N. J. The role of the hippocampus in flexible cognition and social behavior. Frontiers in human neuroscience, 8, 742 https://doi.org/10.3389/fnhum.2014.00742 (2014).

38. Sailani, M. R. et al. DNA-methylation patterns in trisomy 21 using cells from monozygotic twins. PLoS One, 10, e013555 (2015).

39. Sandeen, G., Wood, W. I. \& Felsenfeld, G. The interaction of high mobility proteins HMG14 and 17 with nucleosomes. Nucleic Acids Res, 8, 3757-3778 (1980).

40. Shirakawa, H., Landsman, D., Postnikov, Y. V. \& Bustin, M. NBP-45, a novel nucleosomal binding protein with a tissue-specific and developmentally regulated expression. J Biol Chem, 275, 6368$6374(2000)$.

41. Singh, G. P., Ganapathi, M. \& Dash, D. Role of intrinsic disorder in transient interactions of hub proteins., 66, 761-765 (2007).

42. Tao Deng, Y. et al. Valerie Gailus-Durner, Martin Hrabe de Angelis, Michael Bustin, Interplay between $\mathrm{H} 1$ and HMGN epigenetically regulates OLIG1\&2 expression and oligodendrocyte differentiation. Nucleic Acids Res, 45, 3031-3045 https://doi.org/10.1093/nar/gkw1222 (2017).

43. Therneau, T. M. \& Ballman, K. V. What does PLIER really do? Cancer informatics, 6, 423-431 (2008).

44. Ueda, T., Catez, F., Gerlitz, G. \& Bustin, M. Delineation of the protein module that anchors HMGN proteins to nucleosomes in the chromatin of living cells. Mol Cell Biol, 28, 2872-2883 (2008).

45. Uversky, V. N. et al. Unfoldomics of human diseases: linking protein intrinsic disorder with diseases. BMC Genomics, 10 (Suppl 1), S7 (2009).

46. Zhang, S. et al. Epigenetic regulation of REX1 expression and chromatin binding specificity by HMGNs. Nucleic acids research, 47 (9), 4449-4461 https://doi.org/10.1093/nar/gkz161] (2019).

47. Zhu, F. et al. The interaction landscape between transcription factors and the nucleosome. Nature, 562, 76-81 (2018).

\section{Tables}

Table 1. Description of gene data of five encoding human high mobility binding nucleosome group proteins (HMGN). [Source of NCBI https://www.ncbi.nlm.nih.gov/gene/]. 


\begin{tabular}{llcll} 
Gene & ID* & Locus & Gene name \\
\hline HMGN1 & 3150 & $21 \mathrm{q} 22.2$ & High mobility group nucleosome binding domain 1 \\
\hline HMGN2 & 3151 & $1 \mathrm{p} 36.11$ & High mobility group nucleosome binding domain 2 \\
\hline HMGN3 & 9324 & $6 q 14.1$ & High mobility group nucleosome binding domain 3 \\
\hline HMGN4 & 10473 & $6 \mathrm{p} 22.2$ & High mobility group nucleosome binding domain 4 \\
\hline HMGN5 & 79366 & Xq21.1 & High mobility group nucleosome binding domain 5
\end{tabular}

(*). According with the classification of the National Center for Biotechnology Information (NCBI).

Table 2. Mean Z-ratio values of expression for the five human High Mobility Group Nucleosome Binding Human (HMGN) genes in several brain structures of individuals with Down syndrome.

\begin{tabular}{llllllllll} 
Gene & Gene ID & Brain & HIP & CBC & DFC & OFC & V1C & VFC & ITC \\
\hline HMGN1 & 3150 & 2.81 & 3.00 & 2.35 & 2.63 & 2.07 & 3.00 & 1.86 & 2.45 \\
\hline HMGN2 & 3151 & 0.06 & 0.23 & 0.13 & 0.54 & 0.05 & -0.62 & 0.68 & 0.99 \\
\hline HMGN3 & 9324 & 2.48 & 1.29 & 1.60 & 1.78 & 1.57 & 2.82 & 2.31 & 1.86 \\
\hline HMGN4 & 10473 & 0.37 & 0.85 & 0.77 & 0.27 & 0.84 & 0.41 & 0.19 & -0.27 \\
\hline HMGN5 & 79366 & 2.65 & 2.18 & 1.60 & 2.49 & 1.13 & 1.49 & 2.53 & 2.88
\end{tabular}

Z-ratio value $>1.96$ means significant gene overexpression in the brain of Down syndrome patients ( $Z$ test alpha 0.05. Two tails) (*). Gene ID and data source were obtained from the information consigned in NCBI GeoDataset of a microarray experiment with registration code of GSE59630. (HIP). Hippocampus; (CBC). Cerebellar brain cortex; (DFC). Dorsolateral prefrontal cortex; (OFC), Orbital prefrontal cortex; (V1C). Primary visual cortex; (VFC). Ventrolateral prefrontal cortex and (ITC). Inferior temporal cortex.

Table 3. Mean values of Z-ratio for the five HMGN genes expressed in different age ranks of the brain of human DS individuals.

\begin{tabular}{lllllll} 
Gene & $16-22$ WG & $0-12 \mathrm{M}$ & $2-10 \mathrm{Y}$ & $12-22 \mathrm{Y}$ & $30-39 \mathrm{Y}$ & $40-42 \mathrm{Y}$ \\
\hline HMGN1 & 0.66 & 1.45 & 1.94 & 1.97 & 2.18 & 1.55 \\
\hline HMGN2 & 2.13 & 0.22 & 0.54 & 0.54 & 0.31 & 0.49 \\
\hline HMGN3 & 1.08 & 3.61 & 2.71 & 2.55 & 2.59 & 2.94 \\
\hline HMGN4 & 4.71 & 1.12 & 1.51 & 1.65 & 1.84 & 1.67 \\
\hline HMGN5 & 0.16 & 1.89 & 0.78 & 1.15 & 0.76 & 0.82
\end{tabular}


(WG). Weeks of Gestation. (M). Months. (Y). Years. Data source were previously consigned in the NCBI GeoDataset of a DNA microarray experiment under the registration code of GSE59630.

Table 4. BioGRID data of the five human HMGN interactions with the nucleosome core histones H2BA, $H 2 A G, H 2 A B, H 3 A$ and $H 4 A$ and with the linker histone $H 1 A$.

\begin{tabular}{lllll} 
Interactor & Interaction & Experimental Evidence & Throughput & Score* \\
\hline HMGN1 & HIST1H4A & Affinity Capture-MS (§) & High & $>0.75$ \\
\hline \multirow{2}{*}{ HMGN2 } & HIST1H2BA & Affinity Capture-MS & High & 0.99 \\
\cline { 2 - 5 } & HIST1H3A & Affinity Capture-MS & High & 0.90 \\
\cline { 2 - 5 } & HIST1H2AG & Affinity Capture-MS & High & 0.77 \\
\hline HIST1H1A & Proximity Label-MS (§§) & High & $>0.75$ \\
\hline HMGN & HIST1H4A & Affinity Capture-MS & High & $>0.75$ \\
\hline HMGN4 & HIST1H2AG & Affinity Capture-MS & High & 0.92 \\
\cline { 2 - 5 } & HIST1H3A & Proximity Label-MS & High & $>0.75$ \\
\cline { 2 - 5 } & HIST2H2AB & Affinity Capture-MS & High & 0.88 \\
\hline HMGN5 & HIST1H4A & Affinity Capture-MS & High & $>0.75$
\end{tabular}

* The cut-off threshold is $>0.75$. Data from BioGRID (https://thebiogrid.org/)

(§). Affinity Capture-MS interaction is inferred when a bait protein is affinity captured from cell extracts by either polyclonal antibody or epitope tag and the associated interaction partner is identified by mass spectrometric methods.

(§§). Proximity Label-MS interaction is inferred when a bait-enzyme fusion protein selectively modifies a vicinal protein with a diffusible reactive product, followed by affinity capture of the modified protein and identification by mass spectrometric methods, such as the BioID system.

\section{Figures}




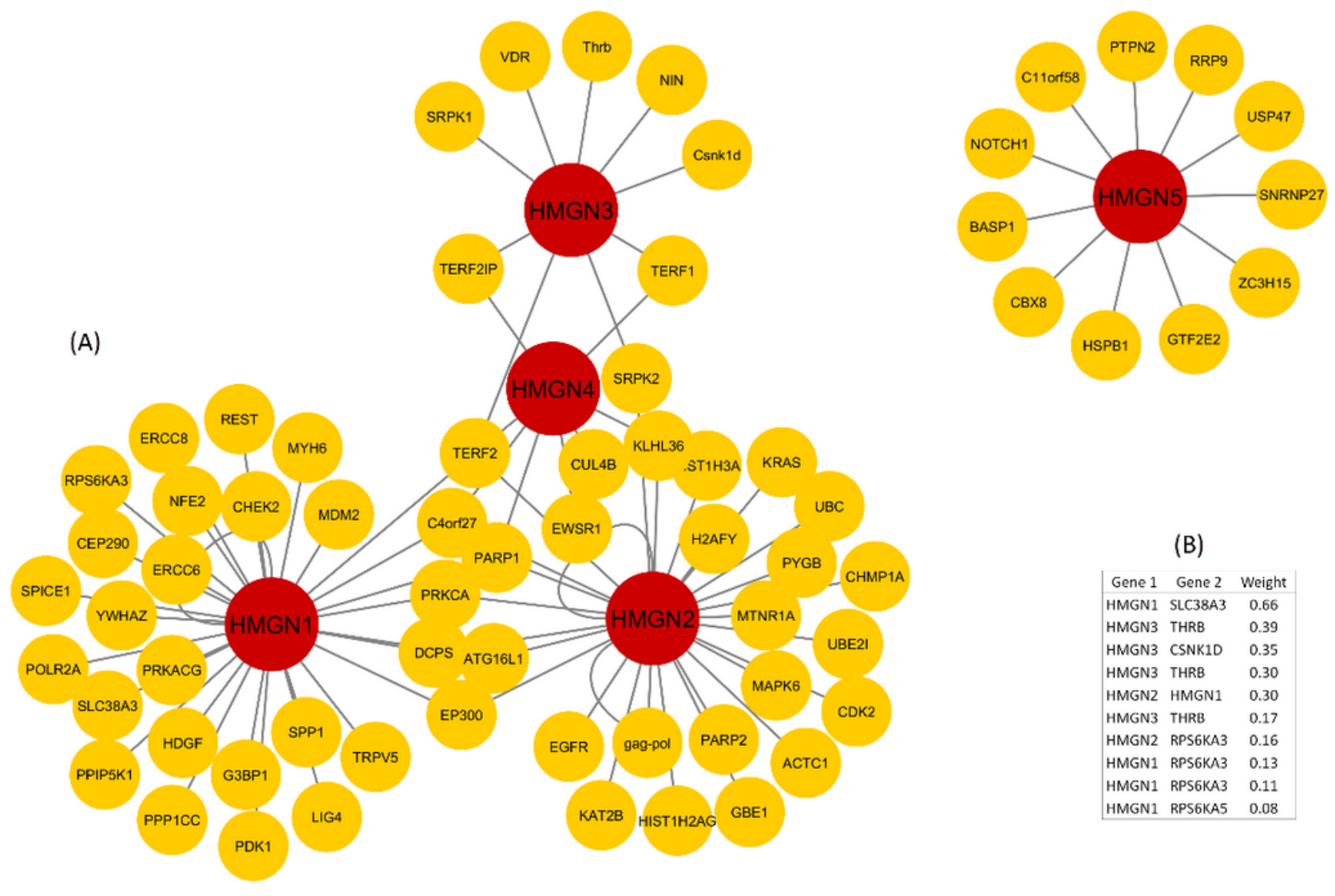

\section{Figure 1}

Protein to protein interaction network between the five human HMGNs and several cellular human genes. (A). Topological structure of the network using Cytoscape program. (B). The statistically significant cellular genes that interact with some of one HMGN. Data were obtained from GeneMania database. 